\title{
Preliminary tests of nano-crystallization of amorphous magnetic ribbons under the influence of periodical, interference laser micromachining
}

\author{
R. Ostrowski, ${ }^{1}$ J. Kusiński, ${ }^{2}$ K. Czyż, ${ }^{2}$ A. Rycyk, ${ }^{1}$ A. Sarzyński, ${ }^{1}$ W. Skrzeczanowski, ${ }^{1}$ M. Strzelec, ${ }^{* 1}$ and O. Czyż ${ }^{2}$

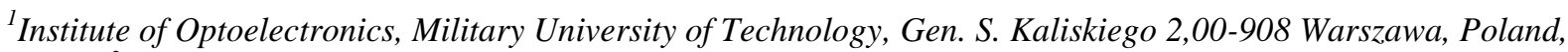 \\ ${ }^{2}$ AGH University of Science and Technology, Al. Adama Mickiewicza 30, 30-059 Kraków, Poland.
}

Received August 29, 2017; accepted September 28, 2017; published September 30, 2017

\begin{abstract}
The main problem connected with crystallization of amorphous magnetic materials from the $\mathrm{FeSiB}(\mathrm{X})$ group is the increase of their fragility with an increasing content of the crystalline phase. To overcome this defect the possibilities offered by the interference, pulse laser heating of $\mathrm{FeSiB}$ ribbons were utilized. A simple interference prismatic system at the output of Nd:YAG laser (8-10ns, 2J, 1064nm) was used. The investigations included the influence of laser radiation energy density, number of laser pulses and their periodical spatial surface arrangement on the crystallization process.
\end{abstract}

Partially de-vitrified $\mathrm{Fe}-\mathrm{Si}$-B based amorphous alloys show enhanced soft magnetic properties due to the nanocrystals embedded in an amorphous matrix, leading to optimum properties [1-2]. Such partial devitrification can be achieved by furnace annealing of the amorphous melt-spun ribbons for various temperature-time combinations. However, usually due to a substantial growth of the nanocrystals and the dependence of coercivity on the grain size, the soft magnetic properties are degraded. On the other hand, the authors of some recent papers have studied rapid annealing using specifically designed furnaces [3] as well as laser processing [4-6] for devitrification of amorphous soft magnetic ribbons. Even if the observed general trends of fast devitrification can be comparable to those observed in furnace annealing, there is a refinement in the size of the $\alpha-\mathrm{Fe}(\mathrm{Si})$ nanocrystals in the rapidly annealed samples [3].

It has been proposed to use Direct Laser Interference Lithography (DLIL) as a pulse annealing process. DLIL is increasingly applied for periodical shaping of material surfaces and for changing their properties [7] and has been previously tested by authors in several biotechnological applications, e.g. [8-9]. The adopted experimental stand (Fig. 1) comprises an Nd:YAG laser oscillator with a stable resonator and a Q-switch modulator, single-channel laser amplifier, a set of harmonic converters and a prism interferometer. The output energy from the oscillator is $30 \mathrm{~mJ}$, and the pulse duration is $8-10$ ns. The inserted diaphragm assures generation of the fundamental transverse mode. After

\footnotetext{
${ }^{*}$ E-mail: marek.strzelec@ wat.edu.pl
}

leaving the oscillator, the laser pulse is amplified in a set of two amplifiers, which results in a maximum output energy of $1 \mathrm{~J}$. A harmonics subassembly, which is not used in the presented experiments, converts the fundamental $\mathrm{Nd}$ :YAG laser wavelength to the wavelengths of $532 \mathrm{~nm}$ and $355 \mathrm{~nm}$, allowing for a decrease in the period of the structures by two or three times. The negative lens forms a divergent laser beam, and the prism, positioned after the lens, divides the beam and projects the interference pattern on the sample. The change of the sample position reflects in the change of the structure period.

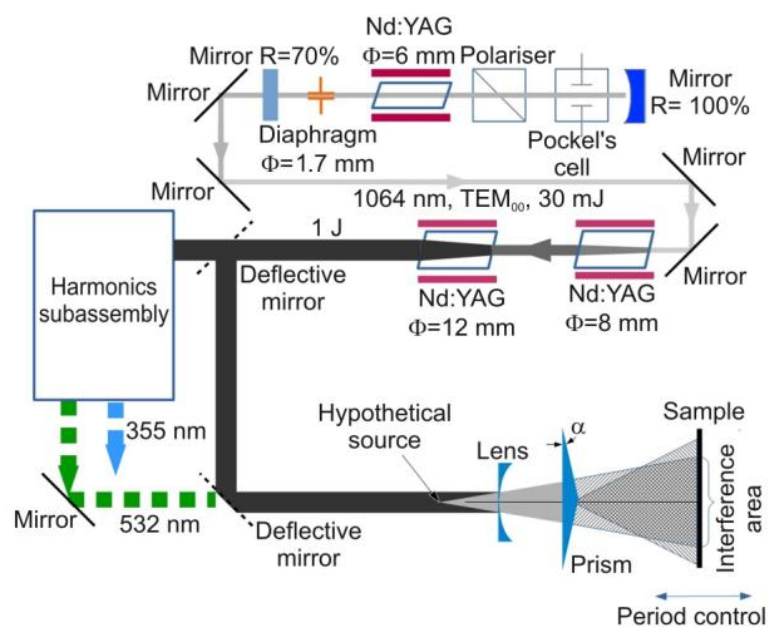

Fig. 1. Scheme of the experimental arrangement based on a prism interferometer.

Interference processing of $\mathrm{FeSiB}$ ribbon (Fig. 2) was performed using a pyramidal tetragonal prism. Properly selected pulse energy and good quality of laser beam are confirmed by the lack of so-called "surface hot points" and areas of over- or under-exposure (Fig. 2a). The drops of a melted and solidified material that are visible in the pictures testify the redeposition of a vaporized material. This phenomenon is characteristic of nanosecond pulse ablation. Moreover, it has been observed that it is much easier to micro-machine a homogeneous structure using 
four beams from prismatic optics than by means of direct lithography based on splitting the wave front (MachZehnder interferometer).

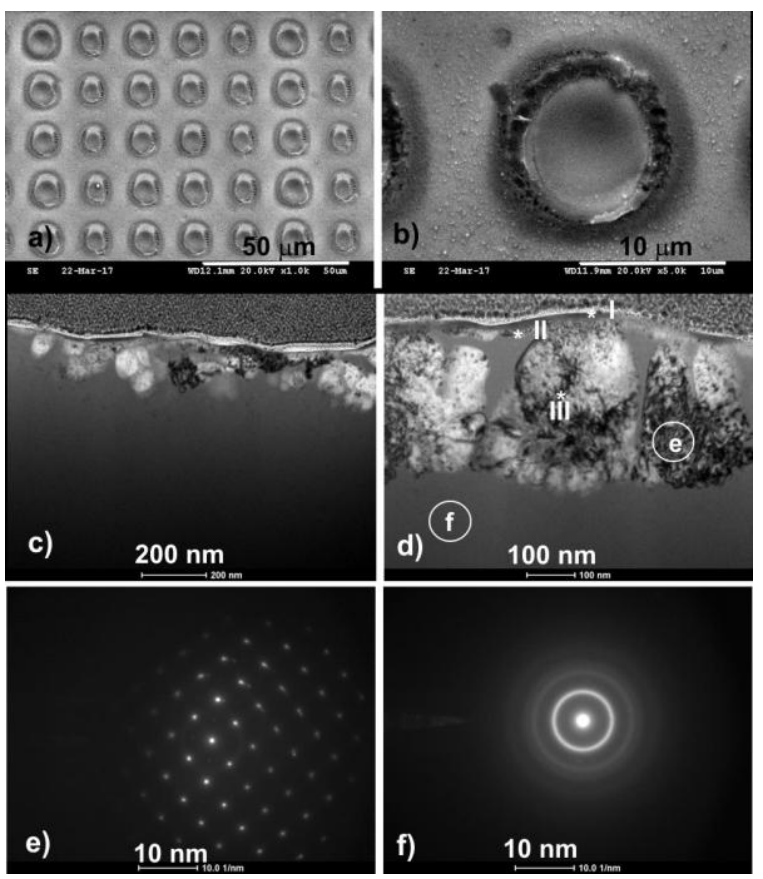

Fig. 2. a, b) SEM images showing surface structure of FeSiB ribbon after laser interference heating (120mJ, 500 pulses); c, d) TEM images showing structure of thin foil extracted using FIB technique from a single heated area (dot) in the transverse cross-section ( $\mathrm{c}-$ border, $\mathrm{d}-$ central part); e, f) - electron diffractions recorded in e) and f) areas indicated in (d); e) from grain with [001] $\alpha$ orientation and f) from amorphous base.

As it can be seen in Fig. 2 a, b), the laser-interference heated ribbon surface is characterized by very homogeneous distribution of laser-affected micro-areas (10 $\mu \mathrm{m}$ dots). The central part of the dot (Fig. 2b) was melted and partially evaporated, while the peripherial zones were heated to the temperature ranges between "liquidus" and "solidus" (dark) and below "solidus" (grey). The area between the dots remained structurally unchanged. TEM images (Fig. 2 c-f) show the cross section of the structure of the thin foil from a single heated area (dot), obtained using the FIB (Focused Ion Beam) technique. The images recorded at the border (c) and central part (d) of the annealed area show that a thin layer of the melted zone, characterized by amorphous structure, can be found near the surface (zone I). A narrow zone of nanocrystal zone II is placed under zone I. The subsequent area is a nanocrystal structure with a tendency to create microdendrites with individual nanometric arms (zone III). The crystal structures of grains in zone III are confirmed by electron diffraction measurements (Fig. 2e). The rest of the FeSiB ribbon is still amorphous, which is confirmed by characteristic ring-shaped electron diffraction (Fig. 2f). A precise analysis of TEM microphotographs (Fig. 2 c,d) demonstrated that $\alpha-\mathrm{Fe}(\mathrm{Si})$ crystals with dimensions of around $50 \mathrm{~nm}$ are created by numerous subgrains with dimensions of around $5 \mathrm{~nm}$.
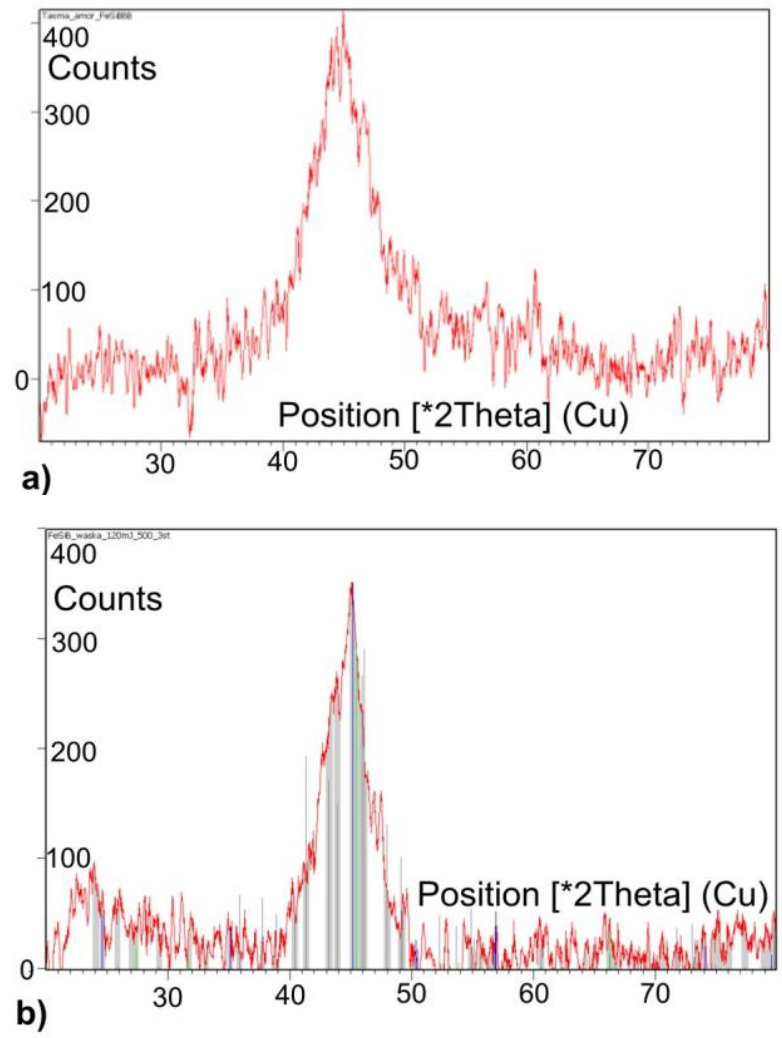

Fig. 3. X-ray diffractograms of Fe-Si-B ribbon: a) in amorphous state; b) after interference laser heating ( $120 \mathrm{~mJ}, 500$ pulses $)$.

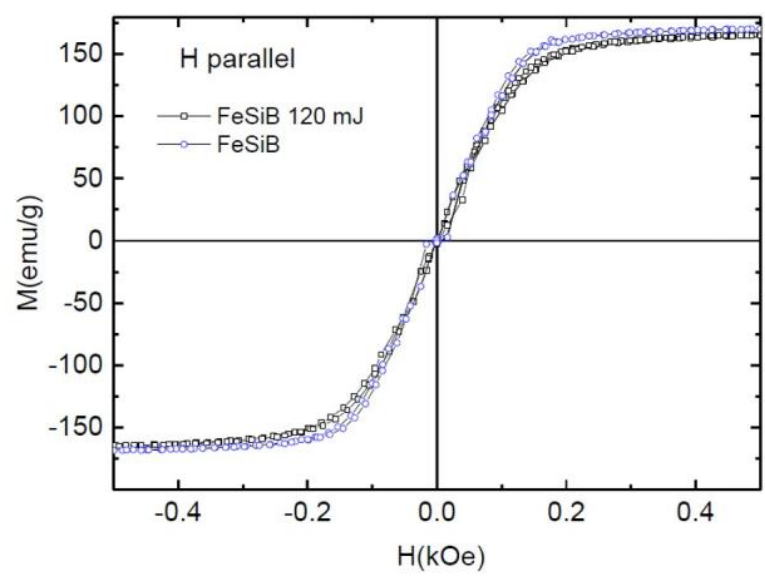

Fig. 4. Results of measurements of hysteresis loops.

The feature that differentiates diffractograms recorded for the ribbon in the initial, as-received, state and after 
interference laser heating was the lower blur of the highest peak $\left(2 \Theta=40-50^{\circ}\right)$ after laser heating (Fig. 3a) than that for the amorphous ribbon (Fig. 3b). Moreover, lines from $\mathrm{FeSi}_{\alpha}$ and $\mathrm{Fe}_{2} \mathrm{~B}$ could be distinguished. The measurement results of the coercive field of samples after laser treatment have shown that it is very similar to the coercive field of amorphous material (Fig. 4).Very similar or even lower is also the value of magnetization $\mathrm{M}$ in comparison with the parameter of the amorphous ribbon.

It has been therefore proven that the devitrification of an amorphous $\mathrm{FeSiB}$ alloy by means of laser interference heating kept the values of the coercive field almost unchanged and magnetization saturation on a high level, characteristic of the amorphous alloy. Both values are important from the perspective of material magnetic losses.

The work that has been performed within the project No 2015/19/B/ST8/01070, "Analysis of factors causing laser interference crystallization of amorphous ribbons made of $\mathrm{FeSiB}(\mathrm{X})$ alloys" was financed by the National Science Centre in Poland (OPUS 10).

\section{References}

[1] Y.R. Zhang, R.V. Ramanujan, Mater. Sci. Eng. A 416, 161 (2006), http://www3.ntu.edu.sg/home/ramanujan/reprints/Ramanujan2006.pdf.

[2] T. Alam, T. Borkar, S. Joshi, S. Katakamb, X. Chenc, N.B. Dahotre, J. Non-Cryst. Solids 428, 75 (2015), http://www.sciencedirect.com/science/article/pii/S0022309315301381.

[3] K.G. Pradeep, G. Herzer, P. Choi, D. Raabe, Acta Mater. 68, 295 (2014), http://www.sciencedirect.com/science/article/pii/S1359645414000482.

[4] S. Katakam, J.Y. Hwang, H. Vora, S.P. Harimkar, R. Banerjee, N.B. Dahotre, Scr. Mater. 66, 538 (2012), http://www.sciencedirect.com/science/article/pii/S1359646211007767.

[5] J. Kusiński, A. Sypień, G. Kusiński, C. Nilson, Mat. Chem. Phys. 81, 390 (2003), http://www.sciencedirect.com/science/article/pii/S0254058403000294.

[6] C. Smith, S. Katakam, S. Nag, X. Chen, R.V. Ramanujan, N.B. Dahotre, R. Banerjee, Mater. Lett. 122, 155 (2014), http://www.sciencedirect.com/science/article/pii/S0167577X14001906.

[7] Ch. Lu, R.H. Lipson, Laser Photonics Rev. 4, 568 (2010), http://onlinelibrary.wiley.com/doi/10.1002/lpor.200810061/full.

[8] K. Czyż, J. Marczak, R. Major, A. Mzyk, A. Rycyk, A. Sarzyński, M. Strzelec, Diam. Relat. Mater. 67, 26 (2016), http://www.sciencedirect.com/science/article/pii/S0925963516300139.

[9] A. Sarzyński, J. Marczak, M. Strzelec, A. Rycyk, K. Czyż, D. Chmielewska, Proc. SPIE, 10159, 101590A (2016), https://www.spiedigitallibrary.org/conference-proceedings-ofspie/10159/1/Laser-micro-structuring-of-surfaces-for-applications-inmaterials-and/10.1117/12.2261785.full?SSO=1. 